\title{
Measurement of muon contents in cosmic ray shower with LHAASO-KM2A around knee region
}

\author{
Hengying Zhang, ${ }^{1, *}$ Cunfeng Feng, ${ }^{1}$ Xiaoting Feng, ${ }^{1}$ Liping Wang, ${ }^{1}$ Huihai He, ${ }^{2,3,4}$ \\ Lingling Ma, ${ }^{2,4}$ Songzhan Chen ${ }^{2,4}$ and Cong $\mathrm{Li}^{2,4}$ on behalf of the LHAASO \\ Collaboration \\ (a complete list of authors can be found at the end of the proceedings) \\ ${ }^{1}$ Institute of Frontier and Interdisciplinary Science, Shandong University, 266237 Qingdao, Shandong, \\ China \\ ${ }^{2}$ Key Laboratory of Particle Astrophyics \& Experimental Physics Division \& Computing Center, Institute \\ of High Energy Physics, Chinese Academy of Sciences, 100049 Beijing, China \\ ${ }^{3}$ University of Chinese Academy of Sciences, 100049 Beijing, China \\ ${ }^{4}$ TIANFU Cosmic Ray Research Center, Chengdu, Sichuan, China \\ E-mail: zhanghy607@mail.sdu.edu.cn, fengcf@sdu.edu.cn
}

\begin{abstract}
The number of muons observed at the ground from air showers is sensitive to the mass composition of cosmic ray and hadronic interaction model. Large High Altitude Air Shower Observatory is a hybrid extensive air shower array and the KM2A is a sub-array covering an area of $1 \mathrm{~km}^{2}$, consisting of electromagnetic detectors and muon detectors, can measure the muon content and shower size of the air shower simultaneously with high precision for cosmic rays in the knee region. The muon detector of $\mathrm{KM} 2 \mathrm{~A}$ is the most powerful muon detector in the current cosmic ray observatory on the ground. We made a detailed comparison of experimental and simulated data. The simulation does for EPOS model.
\end{abstract}

$37^{\text {th }}$ International Cosmic Ray Conference (ICRC 2021)

July 12th - 23rd, 2021

Online - Berlin, Germany

\footnotetext{
${ }^{*}$ Presenter
} 


\section{Introduction}

Muons are created in non-electromagnetic decays of shower hadrons, such as charged pions and kaons. Once produced, muons decouple immediately from the extensive air shower (EAS) and travel almost in straight lines to the detector with smaller attenuation than that for electromagnetic and hadronic particles [1,2]. Studying muons becomes therefore a sensitive and direct way to probe the hadronic physics [3] and to identify possible deficiencies of hadronic interaction models [4, 5]. The muon number in an EAS is also sensitive to the cosmic ray mass composition. So, the number of muons detected by detectors can be used to determine the composition and energy of the primary particles.

This article aims to test the muon contents in EAS with the hadronic interaction model (EPOSLHC). The comparison between experiment and simulation is very necessary because the ground array is used to reconstruct the energy, direction and core position of the primary particles by extending the secondary particle information generated by the EAS. Through the comparison of simulation and experiment, we can better understand the detector performance. The experimental data was collected by muon detector in the LHAASO-KM2A array during 2020. In section 2 briefly introduces the LHAASO-KM2A detector array and introduces the simulation and experimental data. Section 3 is the comparison of experimental and simulation data.

\section{Experiment and simulations}

\subsection{KM2A quarter-array}

The Large High Altitude Air Shower Observatory (LHAASO) is located in Daocheng, Sichuan,

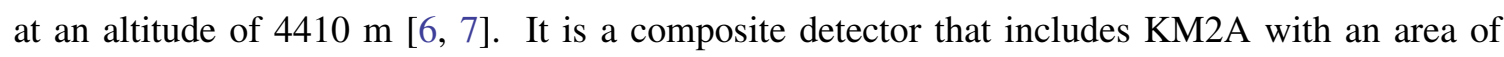
$1.3 \mathrm{~km}^{2}$ and a water Cherenkov detector array (WCDA) with a total area of $78000 \mathrm{~m}^{2}$, and 12 wide-field air Cherenkov/fluorescence telescopes (WFCTAs) as shown in Figure 1. This work uses the KM2A quarter array, which includes 1286 electromagnetic detectors (EDs) and 307 muon detectors (MDs). A layer of $2.5 \mathrm{~m}$ thick soil is overburdened on the MD tank to absorb the secondary electrons/positrons and gamma-rays in air showers [7]. The data in 2020 year are used for this work, for a total live time of 518.63 hours.

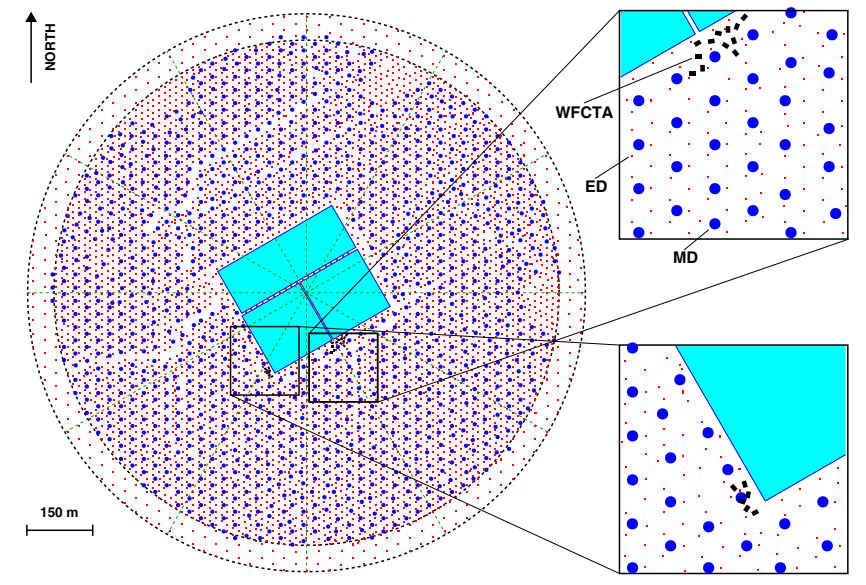

Figure 1: The layout of LHAASO experiment [7]. 


\subsection{Monte Carlo Simulation}

The simulated data is also for KM2A quarter array, using the Cosmic Ray Simulations for KAscade (CORAIKA) [8] software package to simulate EAS. The high-energy hadronic interaction model is EPOS-LHC [9], the low energy hadronic interaction model is FLUKA. The zenith angle range of the shower is 0-70 degrees, and the azimuth angle range is 0-360 degrees with energy spectrum $E^{-2}$ (energy range $10 \mathrm{TeV}-100 \mathrm{PeV}$ ). MC data contain individual sets for different representative primaries: hydrogen $(\mathrm{H})$, helium $(\mathrm{He})$, nitrogen $(\mathrm{N})$, aluminum $(\mathrm{Al})$ and iron $(\mathrm{Fe})$. The parameters are shown in Table 1.

Table 1: Corsika simulation: EPOS-Fluka.

\begin{tabular}{llllll}
\hline \hline Component & $A$ & Energy range $(\mathrm{eV})$ & $\gamma$ & $\theta(\mathrm{deg})$ & $\varphi(\mathrm{deg})$ \\
\hline Proton & 1 & $10^{13} \sim 10^{17}$ & -2 & $0-70$ & $0-360$ \\
$\mathrm{He}$ & 4 & $10^{13} \sim 10^{17}$ & -2 & $0-70$ & $0-360$ \\
$\mathrm{CNO}$ & 14 & $10^{13} \sim 10^{17}$ & -2 & $0-70$ & $0-360$ \\
$\mathrm{MgAlSi}$ & 27 & $10^{13} \sim 10^{17}$ & -2 & $0-70$ & $0-360$ \\
$\mathrm{Fe}$ & 56 & $10^{13} \sim 10^{17}$ & -2 & $0-70$ & $0-360$ \\
\hline \hline
\end{tabular}

To accurately simulate the KM2A detector response, a specific software, G4KM2A [10, 11], was developed in the framework of the Geant4 package (v4.10.00) [12]. The G4KM2A program records the time, position and charge of the secondary particles hitting the detector. The energy, direction and core position of the primary cosmic ray can be obtained by reconstruction. The shower core is uniformity placed within a circle $1000 \mathrm{~m}$ away from the center of the KM2A quarter-array. The number of cosmic ray shower is shown in Table 2. The total number of simulation data is about $1.111 \times 10^{8}$.

Table 2: G4KM2A simulation: the number of cosmic ray shower.

\begin{tabular}{lllll}
\hline \hline Component & $10^{13} \sim 10^{14} \mathrm{eV}$ & $10^{14} \sim 10^{15} \mathrm{eV}$ & $10^{15} \sim 10^{16} \mathrm{eV}$ & $10^{16} \sim 10^{17} \mathrm{eV}$ \\
\hline Proton & $4 \times 10^{7}$ & $4 \times 10^{6}$ & $6 \times 10^{5}$ & $10^{5}$ \\
$\mathrm{He}$ & $10^{7}$ & $10^{6}$ & $10^{5}$ & $2.5 \times 10^{4}$ \\
$\mathrm{CNO}$ & $10^{7}$ & $10^{6}$ & $10^{5}$ & $2.5 \times 10^{4}$ \\
$\mathrm{MgAlSi}$ & $10^{7}$ & $10^{6}$ & $10^{5}$ & $2.5 \times 10^{4}$ \\
$\mathrm{Fe}$ & $4 \times 10^{7}$ & $4 \times 10^{6}$ & $6 \times 10^{5}$ & $10^{5}$ \\
\hline \hline
\end{tabular}

\subsection{Data quality selection}

In order to compare simulation data and experimental data the same reconstruction procedure, and the same selection conditions are used. Simulation and experimental data select the hits within the time window of $[-30,50] \mathrm{ns}$, and the hits of the shower front. 
The operation fetching and reconstruction process of the detector is not completely ideal, and there may be some cases of false trigger and reconstruction error. In order to improve the reconstruction accuracy, the selection criteria are as following:

- $\theta<=30^{\circ}$ : zenith angle of shower less than 30 degrees;

- $D_{r}>65 \mathrm{~m}$ : distance from shower core to array edge;

- NpE1/NpE2>2: NpE1:the number of electromagnetic particles at a distance of $0 \mathrm{~m}$ to $100 \mathrm{~m}$ from the shower core detected by ED; NpE2: the number of electromagnetic particles at a distance of $40 \mathrm{~m}$ to $100 \mathrm{~m}$ from the shower core detected by ED;

- NpE2>25: the number of electromagnetic particles at a distance of $40 \mathrm{~m}$ to $200 \mathrm{~m}$ from the shower core detected by ED;

- $N_{\mu}>10$ : the number of muons at a distance of $40 \mathrm{~m}$ to $200 \mathrm{~m}$ from the shower core detected by MD;

- NtrigE $>50$ : the number of fired EDs;

- $N_{\text {size }}>20000$ : the shower size is reconstructed by fitting the charged particle lateral distribution measured using a modified Nishimura-Kamata-Greisen (NKG) function.

\section{The comparison of simulation and experiment data}

In order to understand the experimental data of the detector and to establish the simulation program, we compare the simulation results with the experimental results.

\subsection{The comparison of unit detector}

The magnitude of hit signal detected by the MD can reproduce the number of muons collected by the unit detector. Using the data after event selection, the event rate of the number of muons $N_{\mu_{-} u}$ detected by the unit MD can be obtained, as shown in the left of Figure 2 and more details about it can be found elsewhere [13]. The single muon peak can also be clearly seen in the distribution, and the muon size spectrum distribution has a curve around 10 particles, which is due to the influence of high-energy electromagnetic particles punch-through effect when they are close to the shower core. As shown in the right of Figure 2, the punch-through effect of high-energy electromagnetic particles is increased when the distance of unit detector to the shower core less than $40 \mathrm{~m}$. Therefore, when counting the number of muons, the detector within $15 \mathrm{~m}$ or $40 \mathrm{~m}$ from the shower core is deducted to ensure the purity of muons.

\subsection{The comparison of KM2A quarter-array}

We compared the experimental and simulated major observational quantities in the KM2A quarter-array as shown in Figure 3. The left of Figure 3 is the distribution of $N_{\text {size }}$ and the right of picture is the distribution of $N_{\mu}$. The simulated data are normalized to the energy spectrum of Gaisser H3a component model [14], and the experimental data are normalized to per second. The 

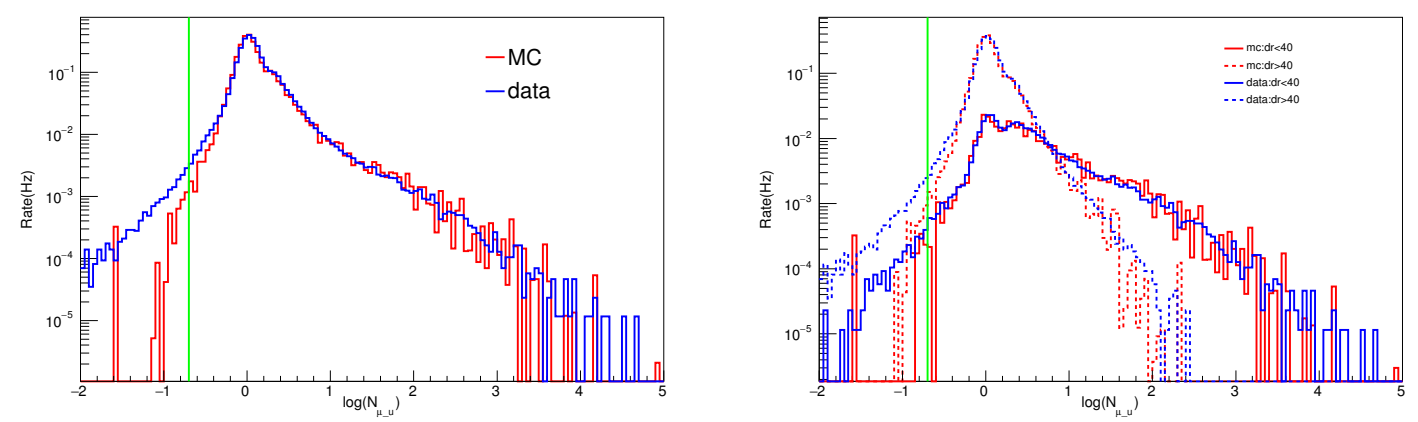

Figure 2: Left: Comparison between MC simulation and experimental data of the daily averaged trigger rate distribution of a typical MD. The horizontal axes indicate the number of particles recorded by these detectors for the triggered events. Detectors with a particle number less than 0.2 , as indicated by the vertical lines, are removed in both MC and experimental data reconstruction [13]. Right: the muon spectrum of the distance of a typical MD to the shower core $(r>40 \mathrm{~m}$ and $r<40 \mathrm{~m})$. The MC simulation (EPOS), with five components, is normalized to the cosmic ray model of Ref.[14].

Figure 3 also shows the ratio of experimental data to simulated data. In a large range, the Ratio $=$ data/MC is a constant, which is about 1.05 . The event rate of experimental data is about $5 \%$ higher than that of simulated data.
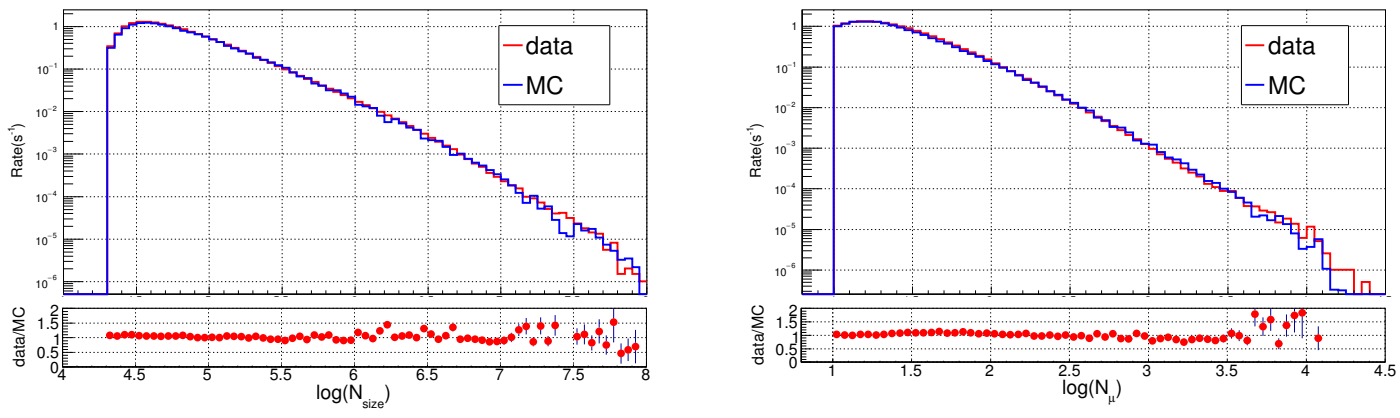

Figure 3: Simulation and experimental distributions of $N_{\text {size }}$ (left), $N_{\mu}$ (right) of KM2A quarter-array.

\section{Conclusions}

In this work, the data of the first KM2A quarter-array are used to measure the shower muon content of the cosmic rays around the knee region. Post-LHC hadronic interaction model is used to describe the development of showers in the atmosphere, and the interactions in the detector are simulated by a G4KM2A procedure. The simulation results are fairly consistent with experimental data. It provides important support for subsequent physical analysis.

\section{Acknowledgements}

This work is supported in China by National Science Foundation of China (NSFC)(No. 11775131, U1931108), Shandong Provincial Natural Science Foundation(No.ZR2019MA014 ) 
and Young Scholars Program of Shandong University (No. 2018WLJH78). The authors would like to thank all staff members who work at the LHAASO site above 4400 meters above sea level year-round to maintain the detector and keep the electrical power supply and other components of the experiment operating smoothly.

\section{References}

[1] L. Cazon, R.A. Vazquez, A .A . Watson, E. Zas, Astrop. Phys. 21 (2004) 71 .

[2] L. Ca- zon, R.A. Vazquez, E. Zas, Astrop. Phys. 23 (2005) 393.

[3] C. Meurer, J. Bluemer, R. Engel, A. Haungs , M. Roth, Czech. J. Phys. 56 (2006) A211 .

[4] A. Aab , et al. , Pierre Auger Coll., Phys. Rev. D 90 (2014) 012012 . erratum: Phys. Rev. D 92 (2015) 019903(E).

[5] A. Aab, et al. , Pierre Auger Coll., Phys. Rev. D 91 (2015) 032003 . erratum: Phys. Rev. D 91 (2015) 059901.

[6] Zhen Cao. Chinese Physics C, 34(2):249-252, jan 2010.

[7] Huihai He. Radiation Detection Technology and Methods, 2, 062018.

[8] D. Heck, et al., 1998, Forschungszentrum Karlsruhe Report No. FZKA 6019.

[9] T. Pierog et al., Phys. Rev. C 92, 034906 (2015).

[10] S. Z. Chen et al., Nuclear Electronics \& Detection Technology 37, 1101-1105 (2017).

[11] S. Z. Chen et al., proceeding of 36th ICRC, (2019).

[12] S. Agostinelli, J. Allison, K. Amako et al., Nuclear Inst. and Methods in Physics Research, A 506, 250 (2003).

[13] F. Aharonian et al 2021 Chinese Phys. C 45025002.

[14] K. Gaisser Thomas, Astroparticle Physics 35, 801-806 (2012). 


\section{Full Authors List: LHAASO Collaboration}

Zhen $\mathrm{Cao}^{1,2,3}$, F. Aharonian ${ }^{4,5}$, Q. An ${ }^{6,7}$, Axikegu ${ }^{8}$, L.X. Bai ${ }^{9}$, Y.X. Bai ${ }^{1,3}$, L.X. Bai ${ }^{9}$, Y.X. Bai ${ }^{1,3}$, Y.W. Bao ${ }^{10}$, D. Bastieri ${ }^{11}$, X.J. Bi ${ }^{1,2,3}$, Y.J. Bi ${ }^{1,3}$, H. Cai ${ }^{12}$, J.T. Cai ${ }^{11}$, Zhe Cao ${ }^{6,7}$, J. Chang ${ }^{13}$, J.F. Chang ${ }^{1,3,6}$, B.M. Chen ${ }^{14}$, E.S. Chen ${ }^{1,2,3}$, J. Chen ${ }^{9}$, Liang Chen $^{1,2,3}$, Liang Chen ${ }^{15}$, Long Chen ${ }^{8}$, M.J. Chen ${ }^{1,3}$, M.L. Chen ${ }^{1,3,6}$, Q.H. Chen ${ }^{8}$, S.H. Chen ${ }^{1,2,3}$, S.Z. Chen ${ }^{1,3}$, T.L. Chen ${ }^{16}$,X.L. $\mathrm{Chen}^{1,2,3}$, Y. Chen $^{10}$, N. Cheng ${ }^{1,3}$, Y.D. Cheng ${ }^{1,3}$, S.W. Cui ${ }^{14}$, X.H. Cuii ${ }^{17}$, Y.D. Cui ${ }^{18}$, B. D'Ettorre Piazzoli ${ }^{19}$, B.Z. Dai ${ }^{20}$, H.L. Dai $^{1,3,6}$, Z.G. Dai ${ }^{7}$, Danzengluobu ${ }^{16}$, D. della Volpe ${ }^{21}$, X.J. Dong ${ }^{1,3}$, K.K. Duan ${ }^{13}$, J.H. Fan ${ }^{11}$, Y.Z. Fan ${ }^{13}$, Z.X. Fan ${ }^{1,3}$, J. Fang ${ }^{20}$, K. Fang $^{1,3}$, C.F. Feng ${ }^{22}$, L. Feng ${ }^{13}$, S.H. Feng ${ }^{1,3}$, Y.L. Feng ${ }^{13}$, B. Gao ${ }^{1,3}$, C.D. Gao ${ }^{22}$, L.Q. Gao ${ }^{1,2,3}$, Q. Gao ${ }^{16}$, W. Gao ${ }^{22}$, M.M. Ge $^{20}$, L.S. Geng ${ }^{1,3}$, G.H. Gong ${ }^{23}$, Q.B. Gou ${ }^{1,3}$, M.H. Gu${ }^{1,3,6}$, F.L. Guo ${ }^{15}$, J.G. Guo ${ }^{1,2,3}$, X.L. Guo ${ }^{8}$, Y.Q. Guo ${ }^{1,3}$, Y.Y. Guo ${ }^{1,2,3,13}$, Y.A. $\mathrm{Han}^{24}$, H.H. He $\mathrm{H}^{1,2,3}$, H.N. He ${ }^{13}$, J.C. He ${ }^{1,2,3}$, S.L. He ${ }^{11}$, X.B. He ${ }^{18}$, Y. He ${ }^{8}$, M. Heller ${ }^{21}$, Y.K. Hor ${ }^{18}$, C. Hou ${ }^{1,3}$, H.B. Hu ${ }^{1,2,3}$, S.

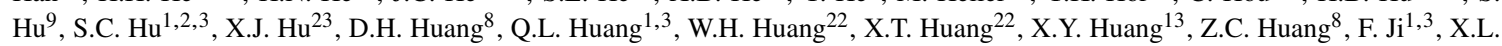
$\mathrm{Ji}^{1,3,6}$, H.Y. Jia ${ }^{8}$, K. Jiang ${ }^{6,7}$, Z.J. Jiang ${ }^{20}$, C. Jin ${ }^{1,2,3}$, T. Ke ${ }^{1,3}$, D. Kuleshov ${ }^{25}$, K. Levochkin ${ }^{25}$, B.B. Li ${ }^{14}$, Cheng Li ${ }^{6,7}$, Cong Li ${ }^{1,3}$,

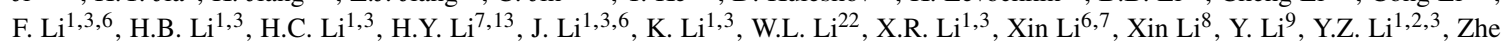

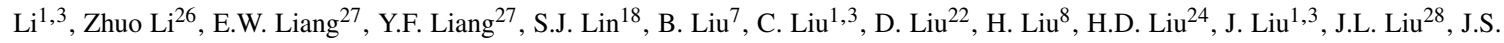

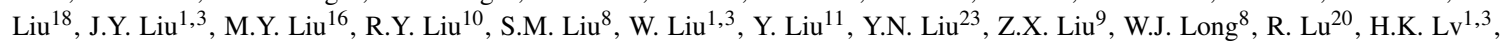

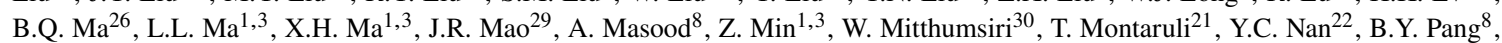
P. Pattarakijwanich ${ }^{30}$, Z.Y. Pei ${ }^{11}$, M.Y. Qi ${ }^{1,3}$, Y.Q. Qi ${ }^{14}$, B.Q. Qiao ${ }^{1,3}$, J.J. Qin ${ }^{7}$, D. Ruffolo ${ }^{30}$, V. Rulev ${ }^{25}$, A. Sáiz ${ }^{30}$, L. Shao ${ }^{14}$, O. Shchegolev $^{25,31}$, X.D. Sheng ${ }^{1,3}$, J.Y. Shi ${ }^{1,3}$, H.C. Song ${ }^{26}$, Yu.V. Stenkin ${ }^{25,31}$, V. Stepanov ${ }^{25}$, Y. Su ${ }^{32}$, Q.N. Sun ${ }^{8}$, X.N. Sun ${ }^{27}$, Z.B.

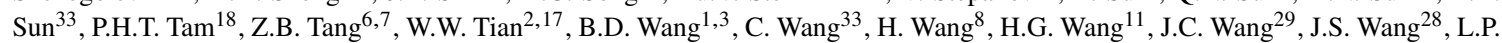
Wang $^{22}$, L.Y. Wang ${ }^{1,3}$, R.N. Wang ${ }^{8}$, W. Wang ${ }^{18}$, W. Wang ${ }^{12}$, X.G. Wang ${ }^{27}$, X.J. Wang ${ }^{1,3}$, X.Y. Wang ${ }^{10}$, Y. Wang ${ }^{8}$, Y.D. Wang ${ }^{1,3}$, Y.J. Wang $^{1,3}$, Y.P. Wang ${ }^{1,2,3}$, Z.H. Wang ${ }^{9}$, Z.X. Wang ${ }^{20}$, Zhen Wang ${ }^{28}$, Zheng Wang ${ }^{1,3,6}$, D.M. Wei $^{13}$, J.J. Wei ${ }^{13}$, Y.J. Wei ${ }^{1,2,3}$, T. Wen ${ }^{20}$, C.Y. $\mathrm{Wu}^{1,3}$, H.R. $\mathrm{Wu}^{1,3}$, S. Wu ${ }^{1,3}$, W.X. Wu ${ }^{8}$, X.F. Wu ${ }^{13}$, S.Q. Xi ${ }^{1,3}$, J. Xia ${ }^{7}, 13$, J.J. Xia ${ }^{8}$, G.M. Xiang ${ }^{2,15}$, D.X. Xiao ${ }^{16}$, G. Xiao ${ }^{1,3}$, H.B. Xiao ${ }^{11}$, G.G. Xin ${ }^{12}$, Y.L. Xin ${ }^{8}$, Y. Xing ${ }^{15}$, D.L. Xu ${ }^{28}$, R.X. Xu ${ }^{26}$, L. Xue ${ }^{22}$, D.H. Yan ${ }^{29}$, J.Z. Yan ${ }^{13}$, C.W. Yang 9 , F.F. Yang ${ }^{1,3,6}$, J.Y. Yang ${ }^{18}$, L.L. Yang ${ }^{18}$, M.J. Yang ${ }^{1,3}$, R.Z. Yang ${ }^{7}$, S.B. Yang ${ }^{20}$, Y.H. Yao ${ }^{9}$, Z.G. Yao ${ }^{1,3}$, Y.M. Ye ${ }^{23}$, L.Q. Yin ${ }^{1,3}$, N. Yin ${ }^{22}$, X.H. You $^{1,3}$, Z.Y. You ${ }^{1,2,3}$, Y.H. Yu ${ }^{22}$, Q. Yuan ${ }^{13}$, H.D. Zeng ${ }^{13}$, T.X. Zeng ${ }^{1,3,6}$, W. Zeng ${ }^{20}$, Z.K. Zeng ${ }^{1,2,3}$, M. Zha ${ }^{1,3}$, X.X. Zhai ${ }^{1,3}$, B.B. Zhang $^{10}$, H.M. Zhang ${ }^{10}$, H.Y. Zhang ${ }^{22}$, J.L. Zhang ${ }^{17}$, J.W. Zhang ${ }^{9}$, L.X. Zhang ${ }^{11}$, Li Zhang ${ }^{20}$, Lu Zhang ${ }^{14}$, P.F. Zhang ${ }^{20}$, P.P. Zhang ${ }^{14}$, R. Zhang ${ }^{7,13}$, S.R. Zhang ${ }^{14}$, S.S. Zhang ${ }^{1,3}$, X. Zhang ${ }^{10}$, X.P. Zhang ${ }^{1,3}$, Y.F. Zhang ${ }^{8}$, Y.L. Zhang ${ }^{1,3}$, Yi Zhang ${ }^{1,13}$, Yong Zhang ${ }^{1,3}$, B. $Z_{\text {Zhao }}^{8}$, J. Zhao ${ }^{1,3}$, L. Zhao ${ }^{6,7}$, L.Z. Zhao ${ }^{14}$, S.P. Zhao ${ }^{13,22}$, F. Zheng ${ }^{33}$, Y. Zheng ${ }^{8}$, B. Zhou ${ }^{1,3}$, H. Zhou ${ }^{28}$, J.N. Zhou ${ }^{15}$, P. Zhou ${ }^{10}$, R. Zhou $^{9}$, X.X. Zhou ${ }^{8}$, C.G. Zhu ${ }^{22}$, F.R. Zhu ${ }^{8}$, H. Zhu ${ }^{17}$, K.J. Zhu ${ }^{1,2,3,6}$ and X. Zuo ${ }^{1,3}$

${ }^{1}$ Key Laboratory of Particle Astrophyics \& Experimental Physics Division \& Computing Center, Institute of High Energy Physics, Chinese Academy of Sciences, 100049 Beijing, China.

${ }^{2}$ University of Chinese Academy of Sciences, 100049 Beijing, China.

${ }^{3}$ TIANFU Cosmic Ray Research Center, Chengdu, Sichuan, China.

${ }^{4}$ Dublin Institute for Advanced Studies, 31 Fitzwilliam Place, 2 Dublin, Ireland.

${ }^{5}$ Max-Planck-Institut for Nuclear Physics, P.O. Box 103980, 69029 Heidelberg, Germany.

${ }^{6}$ State Key Laboratory of Particle Detection and Electronics, China.

${ }^{7}$ University of Science and Technology of China, 230026 Hefei, Anhui, China.

${ }^{8}$ School of Physical Science and Technology \& School of Information Science and Technology, Southwest Jiaotong University, 610031 Chengdu, Sichuan, China.

${ }^{9}$ College of Physics, Sichuan University, 610065 Chengdu, Sichuan, China.

${ }^{10}$ School of Astronomy and Space Science, Nanjing University, 210023 Nanjing, Jiangsu, China.

${ }^{11}$ Center for Astrophysics, Guangzhou University, 510006 Guangzhou, Guangdong, China.

${ }^{12}$ School of Physics and Technology, Wuhan University, 430072 Wuhan, Hubei, China.

${ }^{13}$ Key Laboratory of Dark Matter and Space Astronomy, Purple Mountain Observatory, Chinese Academy of Sciences, 210023 Nanjing, Jiangsu, China.

${ }^{14}$ Hebei Normal University, 050024 Shijiazhuang, Hebei, China.

${ }^{15}$ Key Laboratory for Research in Galaxies and Cosmology, Shanghai Astronomical Observatory, Chinese Academy of Sciences, 200030 Shanghai, China.

${ }^{16}$ Key Laboratory of Cosmic Rays (Tibet University), Ministry of Education, 850000 Lhasa, Tibet, China.

${ }^{17}$ National Astronomical Observatories, Chinese Academy of Sciences, 100101 Beijing, China.

${ }^{18}$ School of Physics and Astronomy \& School of Physics (Guangzhou), Sun Yat-sen University, 519000 Zhuhai, Guangdong, China.

${ }^{19}$ Dipartimento di Fisica dell'Università di Napoli 'Federico II”, Complesso Universitario di Monte Sant’Angelo, via Cinthia, 80126 Napoli, Italy.

${ }^{20}$ School of Physics and Astronomy, Yunnan University, 650091 Kunming, Yunnan, China.

${ }^{21}$ Dèpartement de Physique Nuclèaire et Corpusculaire, Facultè de Sciences, Universitd̀e Gen'eve, 24 Quai Ernest Ansermet, 1211

Geneva, Switzerland.

${ }^{22}$ Institute of Frontier and Interdisciplinary Science, Shandong University, 266237 Qingdao, Shandong, China.

${ }^{23}$ Department of Engineering Physics, Tsinghua University, 100084 Beijing, China.

${ }^{24}$ School of Physics and Microelectronics, Zhengzhou University, 450001 Zhengzhou, Henan, China.

${ }^{25}$ Institute for Nuclear Research of Russian Academy of Sciences, 117312 Moscow, Russia. 
${ }^{26}$ School of Physics, Peking University, 100871 Beijing, China.

${ }^{27}$ School of Physical Science and Technology, Guangxi University, 530004 Nanning, Guangxi, China.

${ }^{28}$ Tsung-Dao Lee Institute \& School of Physics and Astronomy, Shanghai Jiao Tong University, 200240 Shanghai, China.

${ }^{29}$ Yunnan Observatories, Chinese Academy of Sciences, 650216 Kunming, Yunnan, China.

${ }^{30}$ Department of Physics, Faculty of Science, Mahidol University, 10400 Bangkok, Thailand.

${ }^{31}$ Moscow Institute of Physics and Technology, 141700 Moscow, Russia.

${ }^{32}$ Key Laboratory of Radio Astronomy, Purple Mountain Observatory, Chinese Academy of Sciences, 210023 Nanjing, Jiangsu, China.

${ }^{33}$ National Space Science Center, Chinese Academy of Sciences, 100190 Beijing, China. 